

\section{REVISTA \\ TEORÍA Y PRÁCTICA \\ DE LA \\ ARQUEOLOGÍA HISTÓRICA LATINOAMERICANA}

ISSN: 2250-866X (impreso) | ISSN: 2591-2801 (en línea)

AÑo VI, Volumen 6, Verano DE 2017

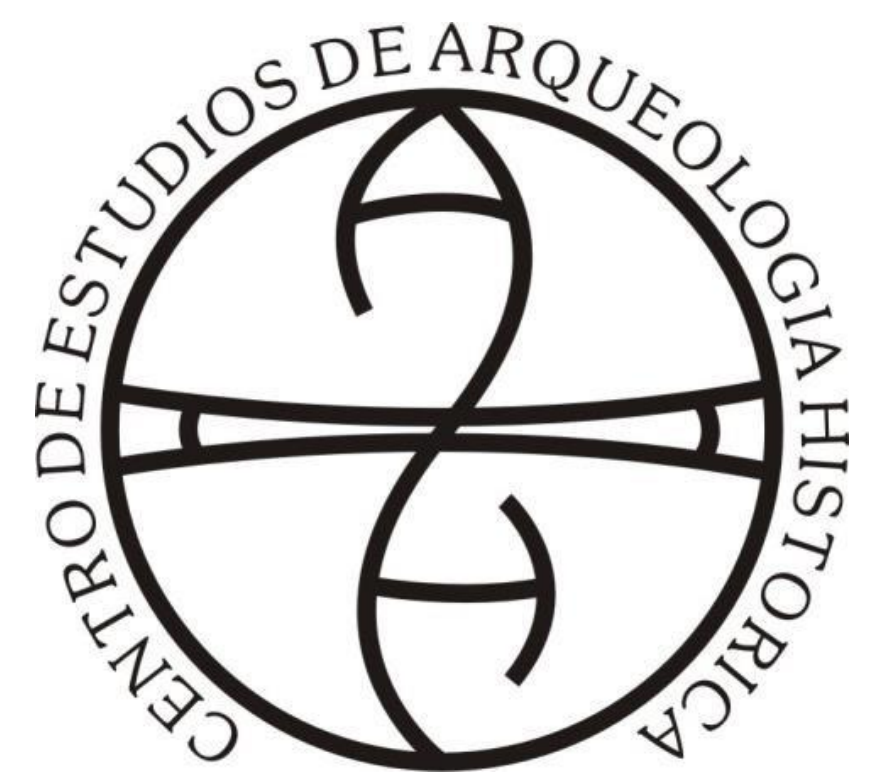

Centro de Estudios de Arqueología Histórica

FACULTAD DE HUMANIDADES Y ARTES | UNIVERSIDAD NACIONAL DE ROSARIO 

PARTICIPA EN LA RED DE ESTUDIOS INTEGRADOS SOBRE LOS PAISAJES SUDAMERICANOS

(Universidad Nacional de Rosario, Universidad Nacional de Río Cuarto,

Universidad Nacional de San Juan, Universidad de la República, Universidad Nacional de Trujillo)

AUTORIDADES DE LA UNIVERSIDAD NACIONAL DE ROSARIO

Rector: Dr. Arq. Héctor Floriani

Vicerrector: Lic. Fabián Bicciré

Secretario general: Lic. Mariano Balla

Secretario académico: Dr. Carlos A. Hernández

\author{
AUTORIDADES DE LA FACULTAD DE HUMANIDADES Y ARTES \\ Decano: Prof. José Goity \\ Vicedecano: Prof. Marta Varela \\ Secretaria Académica: Dra. Liliana Pérez
}

AUTORIDADES DEL CENTRO DE ARQUEOLOGÍA HISTÓRICA

Directora: Dra. Ana María Rocchietti

Secretaria: Prof. Nélida De Grandis

Prosecretaria: Lic. Marianela Biscaldi

Revista del Centro de Estudios de Arqueología Histórica

Teoría y Práctica de la Arqueología Histórica Latinoamericana Actas del Sexto Simposio de Arqueología Histórica Latinoamericana

Presidente del Simposio: Licenciada Mónica Valentini
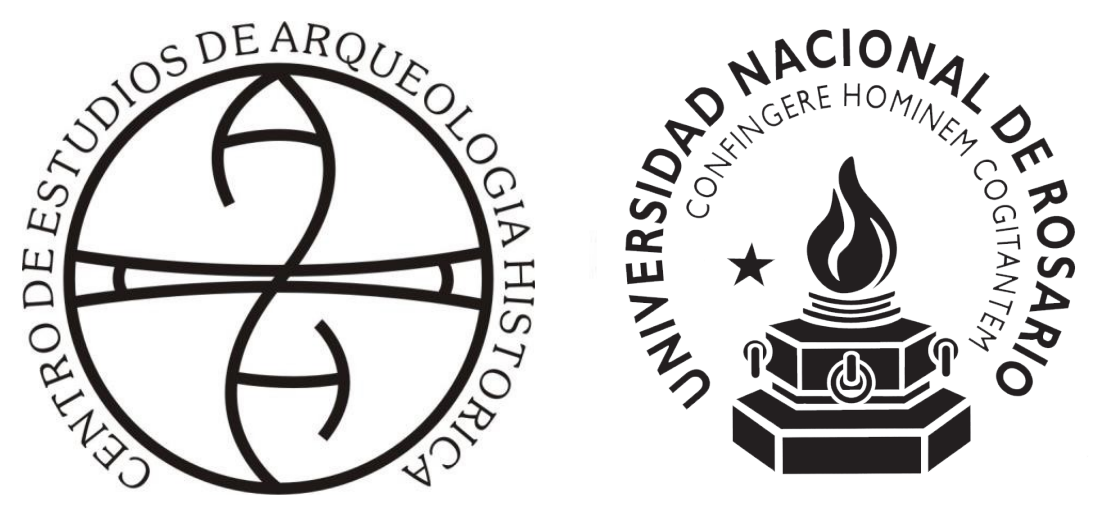


\section{Directoras Editoras}

Ana María Rocchietti y Nélida De Grandis

\section{Secretaria}

Irene Dosztal

\section{Comité Editor}

Ana María Rocchietti, Mónica P. Valentini

Nélida De Grandis, Marianela Biscaldi

Irene Dosztal, María Victoria Roca,

Fátima Solomita Banfi

\section{Comité Científico del V Simposio}

Dra. Nidia Areces

Dr. Raúl Bolmaro

Dr. Luis María Calvo

Lic. Juan Castañeda Murga

Lic. Carlos Ceruti

Dr. Juan Bautista Leoni

Dra. Eugenia Néspolo

Lic. Ruth Adela Poujade

Ing. Adrián Pifferetti

Dr. Mariano Ramos

Dr. Daniel Schávelzon

Dra. Marcela Tamagnini

Dra. Alicia Tapia

\section{Comité Científico}

Dr. Raúl Bolmaro (Universidad Nacional de Rosario)

Dr. Luis María Calvo (Museo Etnográfico de Santa Fe y Parque Arqueológico de Santa Fe La Vieja)

Lic. Carlos Ceruti (CONICET)

Dra. Dora de Grinberg (Facultad de Ingeniería, UNAM)

Dra. Eugenia Néspolo (Universidad Nacional de Luján)

Ing. Tulio Palacios (Comisión Nacional de Energía Atómica)

Lic. Ruth Poujade (Programa Misiones

Jesuíticas - Provincia de Misiones)

Dr. Mariano Ramos (Universidad Nacional de Luján)

Dra. Ana María Rocchietti (Universidad

Nacional de Rosario)
Dr. Daniel Schávelzon (Centro de

Arqueología Urbana - Universidad de

Buenos Aires)

Dra. Marcela Tamagnini (Universidad

Nacional de Río Cuarto)

Dra. Alicia Tapia (Universidad de Buenos

Aires)

Dr. Arno Álvarez Kern (Centro Nacional de

Pesquisas - Brasil)

Dra. Noemí Walsöe de Reca (CONICET)

Lic. Mónica Valentini (Universidad

Nacional de Rosario)

\section{Evaluaron este Volumen}

Horacio Chiavazza, Mónica Valentini,

Nélida De Grandis, Mariano Ramos, Juan

Bautista Leoni, Ana María Rocchietti, Javier García Cano, Flavio Ribero

\section{Diseño y diagramación}

Eugenia Reboiro

(eugenia.reboiro@gmail.com)

\section{Curaduría}

Irene Dosztal y Ana María Rocchietti

Foto de tapa: botellas de vidrio del texto de Orsini y Padula.

\section{Propietario responsable:}

Universidad Nacional de Rosario

Facultad de Humanidades y Artes,

Centro de Estudios de Arqueología

Histórica.

Entre Ríos 758. Rosario, Provincia de Santa

Fe (2000). Argentina.

Telf.: +54 (0341) 4802670

E-mail: ceahunr@gmail.com

Decreto Ley 6422/57 de Publicaciones Periódicas 


\section{Índice}

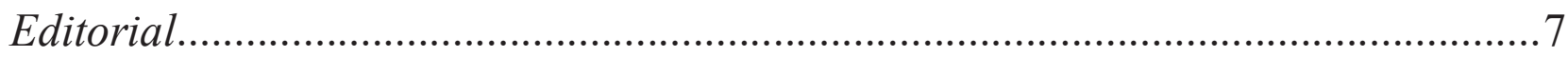

Experimentación, transferencia tecnológica y conservadurismo: notas sobre la introducción del aforro de cobre en los barcos de madera del siglo XVIII......

Nicolás C. Ciarlo

Arqueología social latinoamericana y los desafios de la arqueología urbana.

Ana María Rocchietti

"No todo fue hispánico en la casa de los Larreta". Intervención arqueológica en el jardín del Museo Larreta.

Ricardo Orsini y Horacio Padula

La construcción con ladrillo asentado en barro. El caso de la ciudad de

Rosario

Adrián A. Pifferetti

“Mientras las gárgolas nos miran”. Un sitio arqueológico urbano ¿y religioso?

en la ciudad de Luján. Provincia de Buenos Aires.

Mariano Ramos, Alejandra Raies y Matías Warr

Asentamientos europeos en la cuenca del Río de la Plata: primeras

aproximaciones a Corpus Christi.

Fernando Balducci, Julieta Sartori, Paula Galligani, Fernando Roggero, Macarena Riberi y Abril Castagnola

"De lugares y objetos". La visibilización del pasado afro en el norte de Argentina. Caso de estudio: la ciudad colonial de San Miguel de Tucumán.... 79

Luciana Chávez

Teledetección espacial aplicada a la búsqueda de restos de la Estancia San Miguel del Carcarañá.

Fabián Bognanni, Mónica P. Valentini, Mariano Darigo, Matías Warr y María Lara Moschetoni 
Rescate arqueológico. Hotel Pensión Europea. Posadas.

Misiones. Argentina.

Marianela Biscaldi y Lorena Salvatelli

El Fuerte Independencia revive tres siglos después en medio de un debate sobre sus túneles

Julio Fabián Merlo y María del Carmen Langiano

Estudio de muestras metálicas de vado de Las tres cascadas, Río Cuarto, Córdoba

Adrián A. Pifferetti

Clasificación de botones Prosser y su potencial como indicador

cronológico. Arqueología urbana de Rosario (sitio La Basurita)

María Fernanda Bruzzoni y Sandra Escudero

Una casa del siglo XX y un fuerte del siglo XVI. Arqueología

histórica en Cobos.

Jorge Esteban Cabral Ortiz y Rosana Jimena Flores

Un fechado intrigante

Mario Silveira y Mónica Patricia Valentini

Criterios adoptados para la conservación de los restos de estructura naval del Pecio Zencity de Puerto Madero (Buenos Aires, Argentina): ¿¿RE-ENTERRAR PARA CONSERVAR?!.

Eva Tavella 


\title{
"DE LUGARES Y OBJETOS". \\ LA VISIBILIZACIÓN DEL PASADO AFRO EN EL NORTE DE Argentina CASO de eStudio: la ciudad colonial de San Miguel de TuCumán
}

\author{
Luciana Chávez*
}

\begin{abstract}
Resumen
La presencia de esclavos africanos en la Argentina continúa siendo un tema en discusión y continua elaboración. Negada, silenciada y en el mejor de los casos minimizada dentro de los discursos oficiales de la configuración de la nación, esta ausencia fue tal que la misma se trasladó y arraigó hasta en la propia memoria e imaginario colectivo de los argentinos En este caso presentamos un avance de las tareas de investigación realizadas hasta el momento, en el marco del proyecto de maestría en Memoria Social y Patrimonio Cultural. El mismo presenta una reflexión acerca de cómo determinados espacios y/o lugares, así como diversos acervos documentales relacionados con la esclavitud, al ser reconocidos como parte integrante del patrimonio histórico de los afros argentinos, pueden actuar como herramienta de visibilización aportando al reconocimiento de un pasado silenciado, contextualizando nuestra área de estudio en la ciudad colonial de San Miguel de Tucumán.
\end{abstract}

Palabras clave: Esclavos africanos, patrimonio, San Miguel de Tucumán

\begin{abstract}
The presence of enslaved African people in the South American nation of Argentina continues to be a continually evolving topic of discussion. Denied, silenced, and in the best of cases, minimized within official national discourse regarding Argentine history, these people's stories have even become excluded from the collective memory and worldview of the majority of Argentines. In this particular case we present developments in current research on the subject, carried out within the context of the Master's Program in Social Memory and Cultural Heritage. This specific case reflects on how certain spaces, places, and document archives pertaining to slavery, once they are recognized as forming part of the historical heritage of Afro-Argentines, act as tools to achieve visibility and recognition of a silenced past. The study takes place within the context of the colonial city of San Miguel de Tucumán in the northern Argentine province of Tucumán.
\end{abstract}

Keywords: Africans slaves, Heritage, San Miguel de Tucuman city

\section{Introducción}

El imaginario de identidad argentina se ha caracterizado durante mucho tiempo por resaltar y vanagloriar como privilegio un origen y/o descendencia europea, minimizando otros grupos socia-

\footnotetext{
* Becaria CAPES, Programa de Posgraduación em Memoria Social y Patrimônio Cultural, Universidad Federal de Pelotas. Rio Grande do Sul, Brasil.luchavez23@gmail.com
} 
les, como el caso del indígena, y ocultando deliberadamente la negritud dentro de la configuración nacional.

Sin embargo, tal como lo prueba la extensa documentación histórica como así también el registro material producto de los aportes de la arqueología histórica (Schávelzon, 1998, 1999a; 1999b; 2003 y 2007; Ceruti 2004; 2010; 2011; 2012 y 2013, Carrara y de la Penna 2005, entre otros) los "negros" que llegaron y habitaron el país constituyeron un número mucho mayor al que corrientemente se cree, o se quiere reconocer, como así también lo fue su participación e incidencia dentro de los distintos ámbitos de la vida social de las ciudades ${ }^{1}$. De hecho los censos disponibles para finales del siglo XVIII ${ }^{2}$, tanto en la ciudad de Buenos Aires como las ciudades del interior de la Gobernación del Tucumán (actuales provincias de Jujuy, Salta ,Tucumán, Santiago del Estero, Córdoba, Catamarca y La Rioja) evidencian la representatividad de castas africanas y afrodescendientes entre los habitantes registrados.

Esto nos lleva a preguntarnos y a cuestionarnos entonces por que la idea y el imaginario que comparten la mayoría de los argentinos es totalmente diferente. ¿Qué pasó entonces? ¿Por qué este olvido? Sucede que durante el proceso de configuración de la nación argentina los valores por los cuales lucharon las elites dominantes, mucho diferían al de una nación heterogénea y conformada por un crisol de razas y colores. Todo lo contrario, la nación argentina debía crearse en imagen y semejanza a los estándares europeos razón por la cual todo aquello que no representase parte del discurso era silenciado, en este caso su componente Africano. Como señala Goldberg (2012) “...en la construcción de la Argentina civilizada, las raíces africanas fueron negadas y extirpadas junto con todo elemento material e inmaterial que las recordase..." (p.33).

Esta negación de la realidad histórica del afrodescendiente continúo repitiéndose por largo tiempo dentro de la historiografía nacional. Las narrativas obliteradas acerca "del negro", menospreciadas por los meta relatos de la historia oficial formaron parte de una política de negación y reducción de ese otro social conllevando a la naturalización de este olvido impuesto dentro de la memoria colectiva de los argentinos durante generaciones.

Si bien durante mucho tiempo se continuó reproduciendo este silencio, con el devenir de los años y a consecuencia de los cambios en el pensamiento político y académico, hoy en día podemos advertir una nueva generación de profesionales de diversas áreas abocados al estudio de los afro y afrodescendiente en la Argentina a partir de nuevas líneas de investigación, demostrando la importancia de su legado cultural y la significación que los mismos tuvieron en la construcción del país.

Siguiendo estas perspectivas nuestro trabajo presenta una reflexión acerca de cómo determinados espacios y/o lugares, así como diversos acervos documentales relacionados con la experiencia africana, al ser reconocidos como parte integrante del patrimonio histórico de los afros argentinos, pueden actuar como herramienta de visibilización y reconocimiento del pasado africano, en este caso situándonos en lo que respecta a la ciudad colonial de san Miguel de Tucumán. Resaltando, así mismo, la importancia y rol de los bienes materiales, entendidos como agentes activos dentro de los procesos patrimoniales.

\section{El patrimonio como herramienta de visibilización}

Si bien la noción de patrimonio cultural ha sido históricamente ligada a los procesos de formación de los estados nacionales, a modo de sustento material del ideario de la identidad oficial, aquí abordamos el concepto de patrimonio desde otra perspectiva de análisis. Entendiendo al mismo como un medio a partir del cual se puede llegar a una suerte de democratización del conocimiento del pasado, siendo referente material y simbólico no solo de relatos hegemónicos sino también de los discursos por el reconocimiento y reivindicación de las minorías sociales. 
De acuerdo a Tornatore (2009), la maquinaria patrimonial experimentaría en los últimos años una profusión de iniciativas que escaparían cada vez más a los dispositivos institucionales de regulación con el pasado. En palabras del autor: "Una proliferación de causas y portavoces interviniendo en el campo patrimonial que extrapolarían los marcos instituidos por los dispositivos estatales " (Tornatore, 2009, p. 14).

Se produce entonces un fenómeno de desestructuración y de resignificación complejo y diversificado del patrimonio cultural en su condición de referente histórico y social. La noción de patrimonio se emancipa, se desdobla en función de la diversidad cultural, quebrando así con las ataduras ligadas a concepciones hegemónicas. (Machuca, 2005). Tal como señala Néstor García Canclini (1999), frente a una selección que privilegiaba los bienes culturales producidos por las clases homogéneas, se reconoce ahora que el patrimonio de una nación, también está compuesto por los productos de la cultura popular.

En este sentido el patrimonio cultural no se reduce ya a un único elemento común, sino que responde también a las especificidades y necesidades por parte de diversos grupos sociales, incluyendo aquellos que habían sido marginados y olvidados por las narrativas dominantes.

Así se presenta una nueva manera de interpretar al patrimonio cultural en donde las narrativas y repertorios se despliegan, ya no como medio de control y legitimación por parte del Estado, sino como un lugar de contienda, negociación y reivindicación de diversos grupos sociales, siendo en este caso puntual el de los grupos afrodescendientes de Argentina.

\section{La agencia de lo material}

Tal como afirma Appadurai (1991), las cosas no han estado tan divorciadas de la capacidad de actuar de las personas y del poder comunicativo de las palabras. Constantemente los seres humanos pensamos a través de las cosas, involucrando activamente en nuestra vida el entorno material que nos rodea. Sin embargo, rara vez somos plenamente conscientes del potencial de este accionar, es decir de la manera en que la cultura material es capaz de moldear la forma en que las personas actúan, perciben y piensan (Malafouris y Renfrew, 2010).

En oposición a teorías materialistas en donde los artefactos tienen una vida útil finita, es decir son creados, utilizados hasta ser agotados para luego ser simplemente descartados, surge la noción de la biografía de las cosas (Kopytoff, 1986). La misma sostiene que, así como para las personas es posible trazar una biografía psicológica, profesional, familiar, etc., lo mismo sucede para los objetos, pudiendo contar una trayectoria física, técnica, económica y social de los mismos. De acuerdo a esta perspectiva, los objetos son así mismo actores y participes dentro de las relaciones sociales, siendo utilizados como formas de dar significado a la vida de las personas, construyendo y manteniendo identidades sociales (Jones, 2002).

El entender a los objetos desde esta perspectiva "biográfica" permite así mismo pensarlos a partir de su agencia, es decir como entidades que tienen la capacidad de influir en determinados acontecimientos (Gell, 1998). De esta manera los objetos, como actores sociales, adquieren acción social y participan activamente de la dinámica e interacción social, dejan de ser apenas un producto pasivo del comportamiento para pasar a ser un componente fundamental de las acciones simbólicas.

A partir de estas perspectivas acerca de lo material es que abordamos la interpretación acerca de los objetos patrimoniales, entendiendo que dentro de los procesos de activación patrimonial no serán solo los individuos quienes ejerzan un papel activo, sino que será justamente, a partir de determinados objetos que se logrará viabilizar y validar los discursos por el reconocimiento y visibilización del pasado de los afroargentinos. 


\section{Lugares}

La población de esclavos africanos, al igual que el resto de los habitantes de la ciudad colonial de San Miguel de Tucumán, tuvieron sus lugares de vivienda, trabajo, sociabilización e inclusive espacios destinados para su venta y castigo, como fue la plaza principal de la ciudad. De esta manera, uno de los objetivos planteados en nuestra investigación se centra en el reconocimiento e identificación de diversos lugares relacionados a la experiencia de la esclavitud en la ciudad, interpretándolos como parte del patrimonio histórico afro argentino.

Ahora bien ¿Qué es lo que conlleva el pasar a ser considerados como patrimonio histórico? A partir de nuestra interpretación, estos lugares representan rastros materiales que permiten informar sobre un pasado con frecuencia encubierto, demostrando a su vez la importancia y lo cotidiano de la presencia de esclavos africanos en la dinámica colonial de la ciudad. Los espacios así entendidos pasan a ser resignificados como lugares de memoria (Nora, 1984), definidos en una primera instancia como lugares donde se ancla, condensa, cristaliza, refugia y expresa la memoria colectiva. De acuerdo a Nora (1984) la noción de lugares de memoria se expande a toda unidad significativa, de orden material o ideal, de la cual la voluntad de los colectivos humanos o el trabajo del tiempo ha hecho un elemento simbólico memorial de cualquier comunidad.

Dentro de esta perspectiva se hace hincapié sobre la cualidad que tienen los lugares, a partir de su materialidad, para hacernos pensar y reflexionar acerca de algo que está más allá de ellos mismos. De esta manera el grado de importancia de un lugar no estará dado por su tamaño, magnitud o estilo arquitectónico, sino por las acciones que promueven y el carácter dialógico que lo reviste, es decir, en la capacidad de aunar diferentes acciones, narrativas y acontecimientos en un mismo espacio. Así, los lugares posibles de ser considerados como parte del patrimonio histórico afroargentino pueden ser, desde grandes estructuras arquitectónicas hasta pequeñas viviendas o plazas, a primera vista vacía de evidencia material que la relacione con la experiencia de la esclavitud, pero cargada de significado en el momento en que se lo reconoce como tal.

Así mismo la identificación de estos espacios, articulando en ellos la presencia de la población africana, permite generar una visión más amplia acerca de la dinámica de estos sujetos, materializando en cada uno de los lugares las actividades y prácticas que les fueron comunes.

Los lugares, comprendidos de esta manera, dejan de ser percibidos como mero escenario o consecuencia de acciones, constituyéndose como medio a través de los cuales se materializa un pasado negado, contribuyendo a visibilizar y rectificar la presencia de los esclavos africanos en la ciudad.

\section{Acervos documentales}

En lo que respecta a documentos históricos, el acervo documental disponible en el Archivo Histórico de la Provincia (AHT), representa el registro material que evidencia de manera directa la presencia de esclavos africanos en la ciudad.

Cartas de compraventa de esclavos, hipotecas, donaciones, manumisiones, testamentos, inventarios de bienes hasta expedientes judiciales, tanto civiles como criminales, en donde participan esclavos africanos (ya sea como testigos o como acusados) etc. Cada uno de estos documentos, con sus generalidades y particularidades, expresan la realidad africana desde distintas perspectivas, permitiéndonos acceder a un mundo de prácticas y representaciones de otro modo inaprensibles. Si bien estos sujetos no contaban con la posibilidad de expresarse por escrito, es a partir de los indicios registrados en cada uno de estos documentos que se hace posible visualizar aspectos acerca de sus actividades, prácticas sociales, los espacios habitados, ocupaciones, etc.

Aunque resaltamos la importancia de la información que brindan este tipo de fuentes históricas no perdemos de vista las intencionalidades y matices discursivos muchas veces inherentes a cualquier 
clase de escritos, especialmente cuando el objeto de estudio forma parte de un grupo subalterno. Tal como señala Goldberg, "cuando los españoles aceptaron el tráfico de negros, asimilaron junto con él toda una estructura de prejuicios y estigmas con los que caracterizaban la alteridad: salvajes, animales, brutales, desvergonzados, obscenos", (Goldberg, 2012, p. 29) concepciones que con el tiempo, los hispanocriollos naturalizaron y mantuvieron en la creación subjetiva de una identidad social: "el negro". Por lo tanto un análisis crítico de las fuentes nos lleva a interpretarlas también desde su contexto de producción, analizando lo dicho y lo no dicho (Ruffer, 2005) desde el lugar de su enunciación.

Más allá de la importancia de los documentos históricos como fuentes de información y base a partir de la cual se puede narrar gran parte de la historia de la esclavitud, estos revisten de una función social al constituirse como soporte y evidencia material de un pasado y de una memoria que se ha querido olvidar. En este sentido los documentos son interpretados también como parte del patrimonio o legado afroargentino reposando en ellos valores esenciales como la memoria e identidad de un pueblo constituyéndose como herramienta para el conocimiento y sensibilización por parte de la comunidad en general. De allí la importancia y necesidad de su identificación, preservación, acceso y difusión de los mismos.

\section{Consideraciones finales}

El patrimonio constituye una selección de elementos y conocimientos del pasado articulados en una narración que responde a los intereses de grupo determinado y a las circunstancias del presente. Así, la historia que se narra a través del patrimonio señalará los valores y expectativas que deseamos proyectar hacia el futuro.

Así, además de ser un recurso identitario, el patrimonio constituye un instrumento retorico, ideológico y de poder lo cual nos abre el camino para reflexionar acerca de los usos y valores otorgados al mismo. En este sentido el reconocimiento de un patrimonio histórico afroargentino representa la materialización de un discurso social que busca recuperar parte de un pasado negado, obliterado por discursos oficiales, despertando una conciencia al respecto por parte de la sociedad pero sobre todo contribuyendo al reconocimiento de una identidad cultural argentina heterogénea y diversa.

Así, los documentos históricos conjuntamente con los espacios y lugares interpretados como parte del legado patrimonial afroargentino adquieren carácter activo y medio a través del cual el "negro" cobra visibilidad. Al reconocerlos como tales, se materializa en ellos ese pasado olvidado irrumpiendo de manera directa en el presente fomentando, a su vez nuevas formas de reflexión y conciencia crítica a través de un trabajo comprometido, el trabajo que se hace con la memoria como acción , no como objeto (Jelin, 2002).

Son procesos que se desarrollan a lo largo del tiempo e implican un trabajo activo y de reconocimiento para lograr la semantización de los espacios y los objetos materiales. En palabras de Ballart Hernandez (1997) los objetos del pasado son poseedores de mensajes no siempre explícitos y en ocasiones incluso ocultos, agazapados entre los recovecos de su materialidad, se trata de mensajes codificados que pueden aportar luces y conocimiento, pero para poderlos descifrar y leer hay que pasar antes por un aprendizaje.

\section{Notas}

${ }^{1}$ Existe una amplia bibliografía acerca de la temática, tanto históricas, arqueológicas como antropológicas, las cuales, si bien son la base de nuestro trabajo, no citamos a todas aquí debido a que el presente artículo representa un resumen de los avances realizados hasta el momento, no pudiendo desarrollar más contenidos debido al límite de páginas solicitado. 
2 Tomamos como referencia los datos provenientes del informe de Malaspina de 1789. En Bascary 1999.

\section{Referencias}

APPADURAI, A. (1991). La vida social de las cosas. Perspectiva cultural de las mercancías. México: Editorial Grijalbo

BASCARY, A. M. (1999). Familia y vida cotidiana, Tucumán a fines de la colonia. Facultad de Filosofía y Letras. Tucumán: Universidad Nacional de Tucumán.

CARRARA, M. T. y DE LA PENNA, J. (2006). Las pipas de fumar. En: Carrara, M. T. y De Grandis, N. (Comp.) Santa Fe la Vieja, Arqueología de los siglos XVI y XVII. Rosario: Facultad de Humanidades y Artes. Universidad Nacional de Rosario.

CERUTI, C. (2004). Aporte al conocimiento de la 'cultura del Leyes': la colección del museo de Ciencias Naturales y Antropológicas 'Prof. Antonio Serrano', Paraná, Entre Ríos, Argentina. En Austral, A. y Tamagnini, M. (Comp.) Problemáticas de la Arqueología Contemporánea, Actas del XV Congreso Nacional de Arqueología Argentina, Tomo III (p.357-366). Río Cuarto: Universidad Nacional de Río Cuarto. Córdoba. Argentina.

CERUTI, C. (2010). Los esclavos africanos en Santa Fe la Vieja. En Bárcena, J. R. y Chiavazza, H. (Eds.) Actas del XVII Congreso Nacional de Arqueología Argentina. Tomo III (pp. 1011-1016). Mendoza, Argentina: Facultad de Filosofía y Letras Universidad Nacional de Cuyo. INCIHUSA.

CERUTI, C. (2011). Armando el rompecabezas: piezas enteras del Arroyo Leyes (Santa Fe) vinculadas al agua. En Ramos, M., Tapia, A., Bognanni, F., Fernández, M., Helfer, V., Landa, C., Lanza, M., Montanari, E., Néspolo E. y Pineau, V. (Comp.) Temas y problemas de la Arqueología Histórica. Tomo I. (pp. 241-254). Programa de Arqueología Histórica y estudios pluridisciplinarios. Luján, Argentina: Universidad Nacional de Luján.

CERUTI, C. (2012). Avatares de la colección arqueológica del Arroyo Leyes (Depto. Garay, provincia de Santa Fe, Argentina) o la objetividad científica puesta a prueba. En Rodríguez Leirado E. M. y Schávelzon D. (Eds.) Actas del V Congreso Nacional de Arqueología Histórica, Tomo II (pp. 206235) Editorial Académica Española.

CERUTI, C. (2013). Hay un batracio en mi sopa! Un motivo tradicional del Golfo de Guinea (África) en la cerámica de Santa Fe la Vieja, Argentina. Teoría y práctica de la Arqueología Histórica Latinoamericana 2, pp. 27-36.

GARCÍA CANCLINI, N. (1999). Los usos sociales del Patrimonio Cultural. En Patrimonio Etnológico. Nuevas Perspectivas de Estudio. Aguilar Criado E. (ed.) Consejería de Cultura, (pp. 16-33). Junta de Andalucía.

GELL, A. (1998). Art and agency: an anthropological theory. Oxford: Clarendon Press, Disponible en: https:/es.scribd.com/doc/283376428/Alfred-Gell-Arte-y-Agencia-Una-Teoria-Antropologicapdf. Acceso en Abril 2017

GOldBerg, M. (2012). Plaza San Martin y Parque Lezama. Huellas e Identidades. Sitios de Memoria y culturas vivas de los afrodescendientes en Argentina, Paraguay y Uruguay. Tomo I. La ruta del esclavo. UNESCO. pp.32 -37. 
HERNANDEZ BALLART, J. (1997). El patrimonio histórico y arqueológico; valor y uso. Barcelona: Ed. Ariel Patrimonio Histórico.

JELIN, E. (2002). Los trabajos de la memoria. España: Ed. Siglo XXI

JONES, A. (2002). Material culture and material science: a biography of things. Archaeological Theory and scientific practice. Cambridge: Cambridge University Press.

KOPYTOFF, I. (1986). The cultural biography of things: commoditization as process. En Appadurai, Arjun (Ed.) The social life of things: commodities in cultural perspective. Cambridge: Cambridge University Press.

MALAFOURIS L. y RENFREW, C. (2010). The Cognitive Life of Things: Archaeology, Material Engagement and the Extended Mind. En Malafouris, L. \& Renfrew, C., The cognitive life of things. Recasting the boundaries of the mind. McDonald Institute Monographs. University of Cambridge, pp. 1-12.

NORA, P. (1993). Entre Memoria e Historia: a problemática dos lugares de memoria. San Pablo: Projecto Historia.

PRATS, L. (1998). El concepto de patrimonio cultural. Política y Sociedad 27, Madrid p.p 63-76.

RUFER, M. (2005). Historias negadas. Esclavitus, violencia y relacones de poder en Córdoba a fines del siglo XVIII. Ed. Ferreyra editor.

SCHÁVELZON, D. (1998). La cultura material Africana en Buenos Aires: objetos y contextos. Disponible en: http://www.danielschavelzon.com.ar/?p=25_(Acceso marzo 2016).

SCHÁVELZON, D. (1999). La cerámica de la población africana de Buenos Aires y Santa Fe (siglos XVIII y XIX). En Diez Marín, C. (Ed.), Actas del XII Congreso Nacional de Arqueología Argentina, Tomo I, (pp. 501-508). La Plata. Argentina: Editorial de la UNLP

SCHÁVELZON, D. (2003). Buenos Aires Negra. Arqueología Histórica de una ciudad silenciada. Buenos Aires: Ed. EMECE.

SCHÁVELZON, D. (2007). The vanishing people. Archaeology of the African Population in Buenos Aires. En Ogundiran, A.y Falola T. (Eds.), Archaeology of Atlantic Africa and the African Diaspora (pp. 372-383). Indiana, Estados Unidos: Indiana University Press.

SCHÁVELZON, D. (2015). Una pipa afroa excavada em 1928 em Buenos Aires. Su dispersión por el área del rio de la plata. Contribuciones cientificas GAEA. .27, 151-160.

TORNATORE, J. (2013). Le patrimoine culturel immatériel, entre contrôle et émancipation. O patrimônio cultural imaterial, Entre controle e emancipação. Conferência apresentada no 7ème Seminario Internacional en memoria e patrimonio (SIMP), "Convenção do Patrimônio Imaterial: 10 anos depois », Pelotas: UFPEL.

Recibido: 01/05/2017

Aceptado: 16/09/2017 